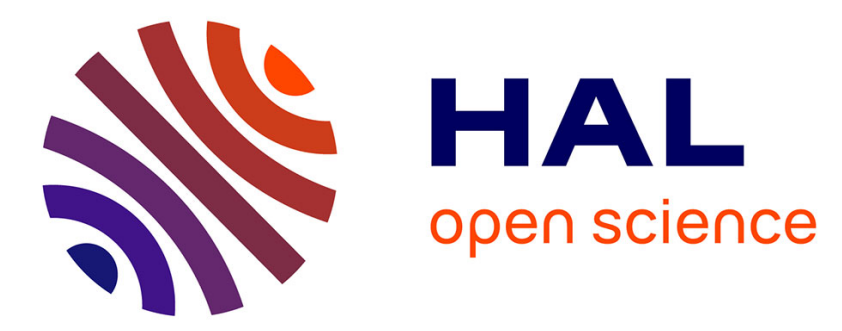

\title{
Ultrasonic imaging of nonlinear scatterers buried in a medium
}

Sylvain Haupert, Guillaume Renaud, Andreas Schumm

\section{To cite this version:}

Sylvain Haupert, Guillaume Renaud, Andreas Schumm. Ultrasonic imaging of nonlinear scatterers buried in a medium. NDT \& E International, 2016, 10.1016/j.ndteint.2016.12.010 . hal-01427158

\section{HAL Id: hal-01427158 \\ https://hal.sorbonne-universite.fr/hal-01427158}

Submitted on 5 Jan 2017

HAL is a multi-disciplinary open access archive for the deposit and dissemination of scientific research documents, whether they are published or not. The documents may come from teaching and research institutions in France or abroad, or from public or private research centers.
L'archive ouverte pluridisciplinaire HAL, est destinée au dépôt et à la diffusion de documents scientifiques de niveau recherche, publiés ou non, émanant des établissements d'enseignement et de recherche français ou étrangers, des laboratoires publics ou privés. 


\title{
Ultrasonic imaging of nonlinear scatterers buried in a medium
}

\author{
Sylvain Haupert ${ }^{\mathrm{a}, *}$, Guillaume Renaud ${ }^{\mathrm{a}}$, Andreas Schumm ${ }^{\mathrm{b}}$ \\ áSorbonne Universités, UPMC Univ Paris 06, CNRS UMR 7371, INSERM UMR S 1146, Laboratoire d'Imagerie Biomédicale, \\ Paris, France \\ ${ }^{b} E D F R \& D$ - Site des Renardières - Avenue des Renardières-Ecuelles Moret sur Loing, France
}

\begin{abstract}
In this paper, an ultrasonic technique for imaging nonlinear scatterers, such as cracks, buried in a medium is proposed. The method called amplitude modulation consists of a sequence of three acquisitions for each line of the image. The first acquisition is obtained by transmitting with all elements of the phased array. Next the second and third acquisitions are obtained by transmitting with odd elements only and even elements only, respectively. An image revealing nonlinear scattering from the medium is reconstructed line by line by subtracting the responses measured with second and third acquisitions (odd elements and even elements) from the response obtained with all elements transmitting. The method was implemented on three different conventional multi-channel electronic platforms equipped with different ultrasonic probes (center frequency $3 \mathrm{MHz}$ to $5 \mathrm{MHz}, 64$ or 128 elements). A crack (6mm-deep x $24 \mathrm{~mm}$-long) in a stainless steel block was investigated. With all probes and multi-channel electronic platforms, higher detection specificity of the crack was obtained with amplitude modulation compared with conventional ultrasound imaging. Image contrast (ratio between crack response amplitude over background amplitude) was increased by $5 \mathrm{~dB}$ with amplitude modulation compared with conventional ultrasound imaging.
\end{abstract}

Keywords: ultrasonic imaging; amplitude modulation; phased array; nonlinear scatterers;

\section{Introduction}

Fast and reliable detection and sizing of cracks is of interest, in particular for hazard monitoring of civil engineering constructions (e.g. dam, bridge, oil pipelines), or for non-destructive testing (NDT) of metallic structures (e.g. railway tracks) and composite structures (e.g. wings of aircrafts). It is well established that cracks produces nonlinear (or wave-amplitude dependent) acoustic scattering. Numerous nonlinear ultrasonic techniques [1-3] have been proposed to detect nonlinear scattering the last couple of decades. Most of them give access to a single nonlinear parameter for the whole structure. Despite the number of studies, only few of them were able to really show a correlation between a global amount of nonlinearity and the density of cracks [4-9] or the length of a buried single crack [10-15]. On the other hand, few techniques were specifically designed to create an image of the nonlinearity and were generally restricted to the surface of the medium [16-21]. Recently, two methods were proposed to image nonlinear scatterers buried in homogeneous materials (e.g. metallic alloys), by the measurement of the sub-harmonics [22] or the nonlinear diffused field [23] using ultrasonic phased array. The first one requires specific and expensive electronic device and probe in order to deliver large energy to activate the crack lips. This technique is also self-restricted to sub-harmonics. It does not take into account other nonlinear responses taking place at the crack (e.g. ultra-harmonics generation, velocity changes, waveamplitude dependent attenuation...). The second technique is promising as it takes into account the entire nonlinear response but requires the acquisition of the diffusive field generated element by element and complicated signal processing which makes it potentially difficult for real time imaging (i.e. maximum 10 images per seconds).

In this paper, we propose to apply a technique based on the so-called Amplitude Modulation (AM) (or power modulation) method [24]. This technique, sometimes combined with the so-called Pulses Inversion [25] (PI) sequence, is routinely used in medical for real-time contrastenhanced ultrasound imaging [26]. It involves contrast agent which contains microbubbles producing nonlinear scattering while soft tissue creates linear scattering. We show that this method can be directly transferred to NDT for the characterization of a crack. The feasibility is demonstrated on a piece of stainless steel 304 with a $6 \mathrm{~mm}-$ deep crack created by thermal fatigue.

\section{Amplitude modulation imaging method}

At the microscale level, "clapping" and "rubbing" of rough surfaces of flaws can cause a distortion of the incident acoustic waves. The distortion has the specific characteristic to be amplitude dependent. In other words, the response of the nonlinear scatterers is not proportional to the vibration amplitude while the response of the linear scatterers (e.g. the signal of the back wall) is. The AM methods are based on this specific characteristic. Among the AM techniques, one can distinguish the Scaling Subtraction Method [27] (SSM) already used in NDT, e.g. for early damage detection in concrete [28] or implant stability monitoring [29]. SSM technique consists in emitting a first signal at low amplitude 
$A$ followed by another identical signal at higher amplitude $k A$, such as nothing has changed except the amplitude of excitation. The nonlinear residual $R_{N L}$ is calculated by subtracted the rescaled low amplitude response (by a factor $k$ ) to the high amplitude response. This technique works with an array transducer as well as with a single element probe, with pulses, coded wave or continuous wave [28,29]. However, SSM is limited by the nonlinearity of the electronics which is scaled too. Based on the know-how in contrast-enhanced ultrasound imaging in medical $[24,26]$, we propose an alternative to SSM which overcomes this problem but requires a phased array probe. It consists of a sequence of three acquisitions (see Figure 1) at the very same focal points $(x, z)$ (i.e. the same delay laws are applied for each acquisition). First, an image of the medium is generated by electronic beam steering (i.e. the probe does not move) with all the elements of the phased array (Transmit amplitude $=A$ ). Then, a second acquisition is obtained by exciting solely the odd elements (Transmit amplitude $=A / 2$ ). Finally a third set of signals is recorded with only the even elements active (Transmit amplitude $=$ $A / 2$ ). Note that the excitation voltage remains the same for each acquisition, only the number of active elements varies. Thus, three sets of signals are recorded each time by all elements of the ultrasonic phased array. After the acquisition process, three waveforms (full; even; odd) are reconstructed from the three sets of received signals. The reconstruction process involves three parts: 1) the three sets of received signals are bandpass-filtered in the range frequencies of the probe; 2) the very same delay law is applied to the three sets of signals; 3) full, even and odd waveforms are obtained by summation of all filtered and shifted signals within each set. The final nonlinear image is then created line by line by subtracting the odd and even waveforms from the full waveform. As a result, the signals produced by linear scatterers are suppressed while the signals produced by nonlinear scatterers remain. Indeed, the linearity of the additivity rule is broken by the nonlinearity present in the medium. This process is then able to selectively detect nonlinear scatterers and eventually produces an image showing the localization of the crack.

A conventional ultrasound image is also reconstructed for comparison.

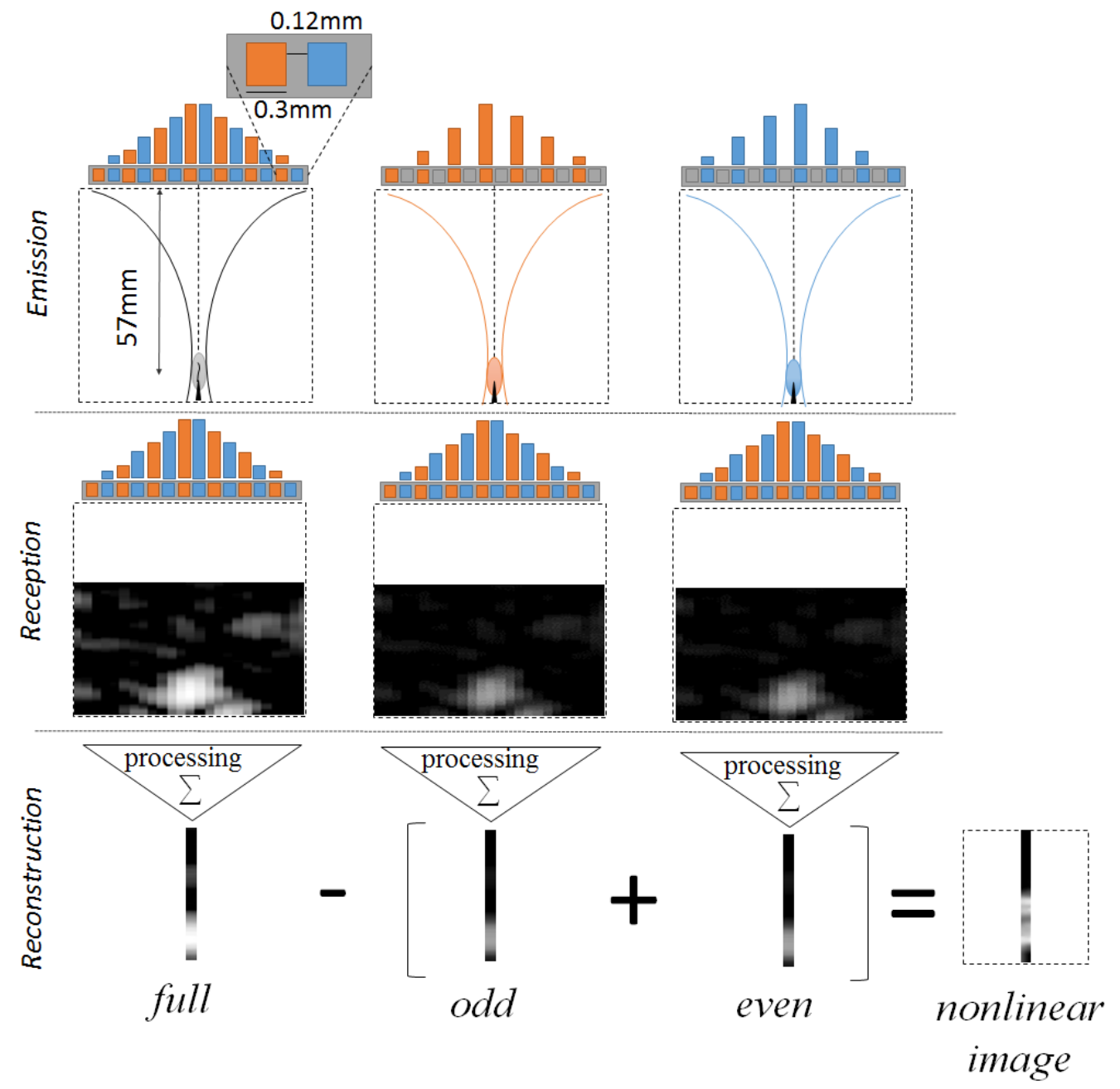

Fig. 1. Schematic of the Amplitude Modulation technique which consists of a sequence of three acquisitions at the very same focal points with alternatively activation of full, odd or even elements. Signal processing consists in bandpass filtering and time shifting signals before summation then subtraction. 


\section{Experimental thermal fatigue crack imaging}

The demonstration of feasibility of the proposed AM method was done on a stainless steel AISI304 parallelepiped sample $(61 * 150 * 100 \mathrm{~mm})$ with a small crack (Trueflaw Ltd, Espoo Finland). The buried crack was produced in-situ by controlled thermal fatigue loading in order to create a thermal fatigue crack with realistic residual stress fields. The flaw grow with thermal fatigue damage mechanism. Consequently, the flaw location is affected by the local material properties. Especially, if the sample contains marked inhomogeneity's or stress risers, the cracks grow in to the natural weakest location. This process is known to produce realistic simulation of mechanical and thermal fatigue cracks and good simulation of stress corrosion cracks [30]. The apparent $24.2 \mathrm{~mm}$ length of the crack was optically measured at the surface of the sample by dye penetrant inspection while the $5.9 \mathrm{~mm}$ depth of the crack was estimated, not measured, by the manufacturer. The compressional wave velocity in the sample is assumed to be $5700 \mathrm{~m} / \mathrm{s}$.

The validation of the method is performed with a 64 elements $3.8 \mathrm{MHz}$ array with an inter-element's pitch of $0.42 \mathrm{~mm}$ and an element's width of $0.30 \mathrm{~mm}$ (Imasonics, Voray sur l'Ognon France) driven by a Lecoeur electronic (Lecoeur, Chuelles, France) [40MHz sampling frequency, $12 \mathrm{bits}, 70 \mathrm{Vpp}$ ]. The transducer array is gel coupled to the opposite surface of the crack. A mechanical translation is performed in nine steps along the crack (i.e direction $y$ ) with the probe positioned centrally over the crack. The scanning step is $5 \mathrm{~mm}$ which corresponds to half of the elevation width of the probe (see Figure 2). For each position, both conventional and nonlinear images are obtained by focusing the ultrasound beam at $57 \mathrm{~mm}$ in depth (i.e direction $z$; where the crack tip is expected) and by beam-steering horizontally (i.e. direction $x$ ) between $-4 \mathrm{~mm}$ to $4 \mathrm{~mm}$ with a $0.25 \mathrm{~mm}$ step.

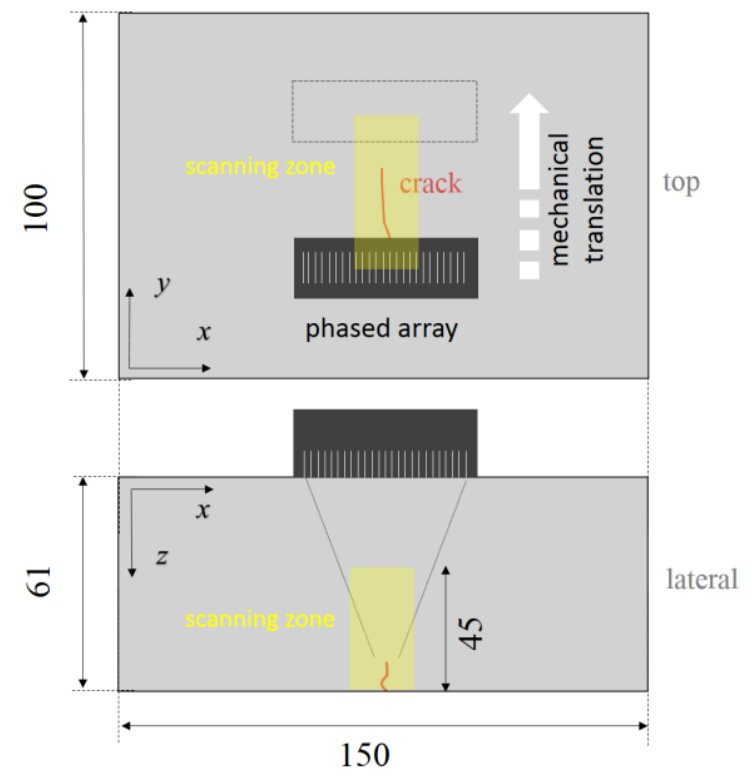

Fig. 2. Schematic of the stainless steel specimen with the location of the phased array and the scanning zone. [dimensions in $\mathrm{mm}$ ]

\section{Results and discussion}

A 3-D representation of the medium is done based on the nine reconstructed slices for the conventional and the AM methods (Figures $3 \mathrm{a}$ and $3 \mathrm{~b}$ ). For the sake of clarity, images are restricted in depth to two third of the sample thickness.

In the first slice (i.e. away from the crack), the back wall echo is the main feature observed in the conventional image (Figure 3d) while the background of the image is dominated by speckle caused by the grains (typically 100-500um) present in the stainless steel. In the nonlinear image (Figure 3f), the back wall does not totally disappear, it is called the nonlinear residue. Indeed, this nonlinear residue corresponds to the remaining linear scatterers that are not completely canceled after applying the amplitude modulation method. To compare both images and quantify the nonlinear residue, they are plotted in a $\mathrm{dB}$ scale, where $0 \mathrm{~dB}$ is defined as the maximum amplitude signal in the conventional image, i.e. the echo from the back wall. The average level of the remaining nonlinear residue visible at the back wall location is $-36 \mathrm{~dB}$, which is close to the interelement coupling of the phased array (information given by the manufacturer). If the inter-element coupling is linear, the cancellation should be perfect. In reality, one can suspect that the inter-elements coupling is not strictly linear which leads to a slight disruption of the linearity requested for a perfect cancelation of signals coming from linear scattering. The average level of the background noise (i.e. corresponding to the mean values in $\mathrm{dB}$ in the region of interest defined by the dash lines in Figures 3c-f) observed in the nonlinear image is $-79 \mathrm{~dB}$. The origin of this noise is not caused by linear scattering due to the grains but is due to electronic and thermal fluctuations. If necessary averaging of multiple reconstructed nonlinear images acquired at the same position would improve the signal to noise ratio between nonlinear scatterers and background noise. 
conventional image

(a)
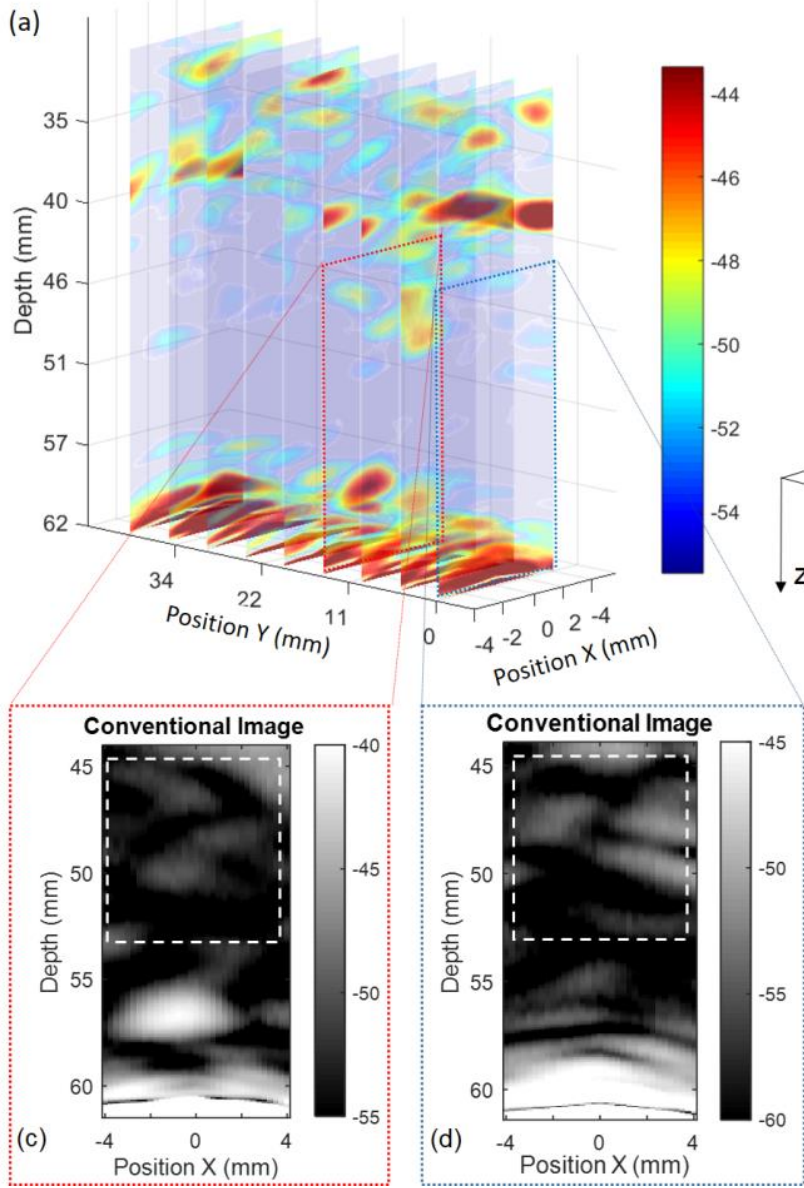

(b)

nonlinear image
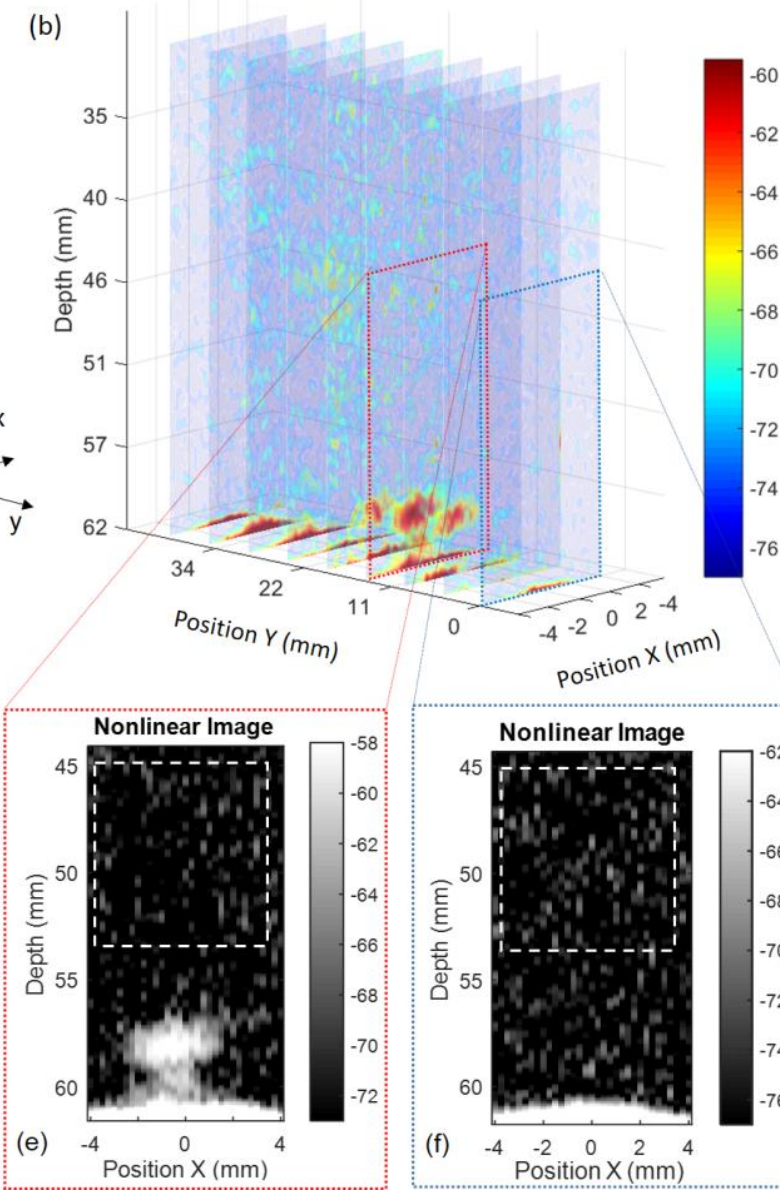

Fig. 3. 3-D representation of the medium obtained by the conventional (a) and the amplitude modulation (b) methods with a $3.8 \mathrm{MHz}-64$ elements phased array. Two slices, at the crack location and outside of the crack, are presented for the conventional method (c, d) and the amplitude modulation method (e f) respectively. The dynamic gray level intensity are $15 \mathrm{~dB}$ and $25 \mathrm{~dB}$ for conventional (c, e) and nonlinear (e, f) images respectively. [intensity is in $\mathrm{dB}$ ]

At the crack location, the conventional and the nonlinear images show both a signal produced by the crack (Figures $3 c$ and $3 e$ ). At a first glance, the crack appears visible and distinguishable in three contiguous slices of the 3-D representation obtained with the AM method. On the contrary, the crack is clearly unraveled in one slice only with conventional imaging. This qualitative analysis is confirmed by quantitative comparisons done on the slice with the largest response of the crack. In the conventional image, the maximum signal due to the linear scattering by the crack is equal to $-40 \mathrm{~dB}$ (figure $3 \mathrm{c}$ ), while the background noise is $54 \mathrm{~dB}$. In the nonlinear image, the maximum response of the crack is equal to $-56 \mathrm{~dB}$ while the background noise remains at $-75 \mathrm{~dB}$, which means a contrast of $19 \mathrm{~dB}$, better than the $14 \mathrm{~dB}$ obtained with the conventional image. Thus, the nonlinear signature of the crack appears to be more specific and more contrasted than the linear signature. Indeed, in the conventional image, the linear response is not specific to the crack (e.g. crack tip) but comes from other linear scatterers, essentially the grains in the stainless steel specimen. This structural noise, also referred to as speckle noise, looks similar to the crack response as they are both the result of the same origin, i.e. acoustic impedance mismatching. The speckle deteriorates the contrast of the conventional images making it difficult to disentangle from the crack echo. On the contrary, the AM method detects selectively the nonlinear response of the crack.

The maximum of the linear response of the crack is located at $56.6 \mathrm{~mm}$ in depth while the maximum of the nonlinear response is located at $57.9 \mathrm{~mm}$. The superposition of both areas (i.e. corresponding to the values greater than the maximum response $-6 \mathrm{~dB}$ in each image) shows little overlapping of conventional and nonlinear areas (orange color in Figure 4). From this image, one can estimate the crack depth to be approximately $6 \mathrm{~mm}$, close to the theoretical $5.9 \mathrm{~mm}$ given by the manufacturer. Moreover, the maximum responses in the two types of images are not colocalized in space. In this particular sample, we can speculate that the conventional imaging is sensitive to the open part of the crack (green color in Figure 4). Since there is no contact between the crack lips, no nonlinear scattering 
is expected. The AM method is sensitive to the (partially-) closed part of the crack (blue color in Figure 4). Indeed, if weak static stress exists on the crack lips, contact elastic nonlinearity such as kissing, clapping or rubbing as well as thermo-elasticity mechanisms can occur [31]. If local static stress at the crack is high, the crack is tightly closed, consequently neither linear scattering nor nonlinear scattering is produced. However the use of nonconventional high-power ultrasound equipment [32] may be able to generate significant nonlinear scattering at this closed part of a crack.

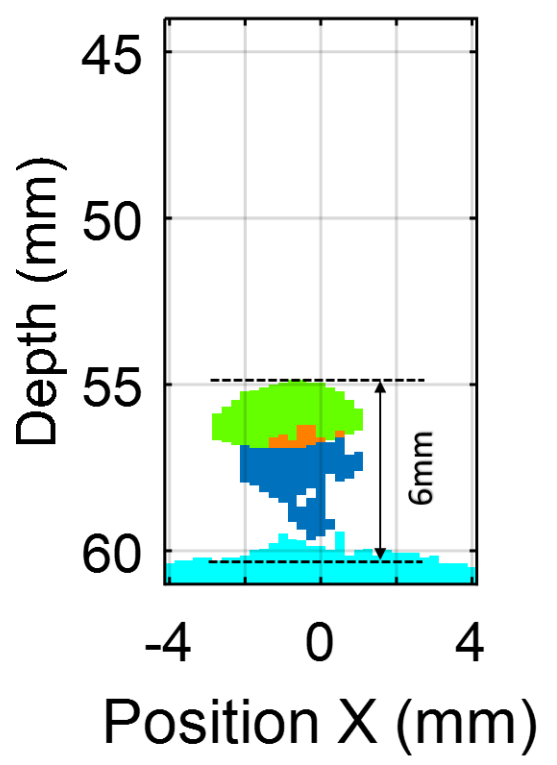

Fig. 4. Superposition of the conventional and nonlinear responses corresponding to the values greater than the maximum response $-6 \mathrm{~dB}$ in each image. Color legend: white is the background / cyan is the back wall / green is the linear response only / blue is the nonlinear response only / orange is when both responses are superimposed.

This very first result was confirmed with two other multi-element probes (from $3 \mathrm{MHz}$ to $5 \mathrm{MHz}, 64$ or 128 elements) and two other multi-channel electronic systems. As an example, one result obtained with a $5 \mathrm{MHz}$ phased array (128 elements - pitch of $0.5 \mathrm{~mm}$ - Imasonics, Voray sur l'Ognon, France) coupled to a MultiX++ system (output voltage $180 \mathrm{Vpp}-\mathrm{M} 2 \mathrm{M}$, Les Ulis, France) is shown in Figure 5. While another result obtained with a different $5 \mathrm{MHz}$ phased array (64 elements - pitch of $1 \mathrm{~mm}$, Imasonics, Voray sur l'Ognon, France) and an OEM-PA system (output voltage $185 \mathrm{Vpp}$ - Advanced OEM Solutions, Cincinnati, USA) with an out is shown in Figure 5. The images are very similar which suggests that the AM method is robust and can be implemented with any conventional ultrasonic equipment. The differences between images is explained by the different probe's aperture and position and the different signal to noise ratio. On can notice that we did not observe nonlinear signals above the crack tip detected by conventional imaging as it was observed with other nonlinear imaging techniques on different specimen with different damage [22,23]. However, we think that the AM method should be able to provide better characterization of the crack, especially for (partially-) closed cracks, invisible to conventional ultrasound. In order to confirm the capability of AM method, other specimens with different defect geometries should be now tested as the nonlinear response intensity may depend on the crack morphology and orientation [15].
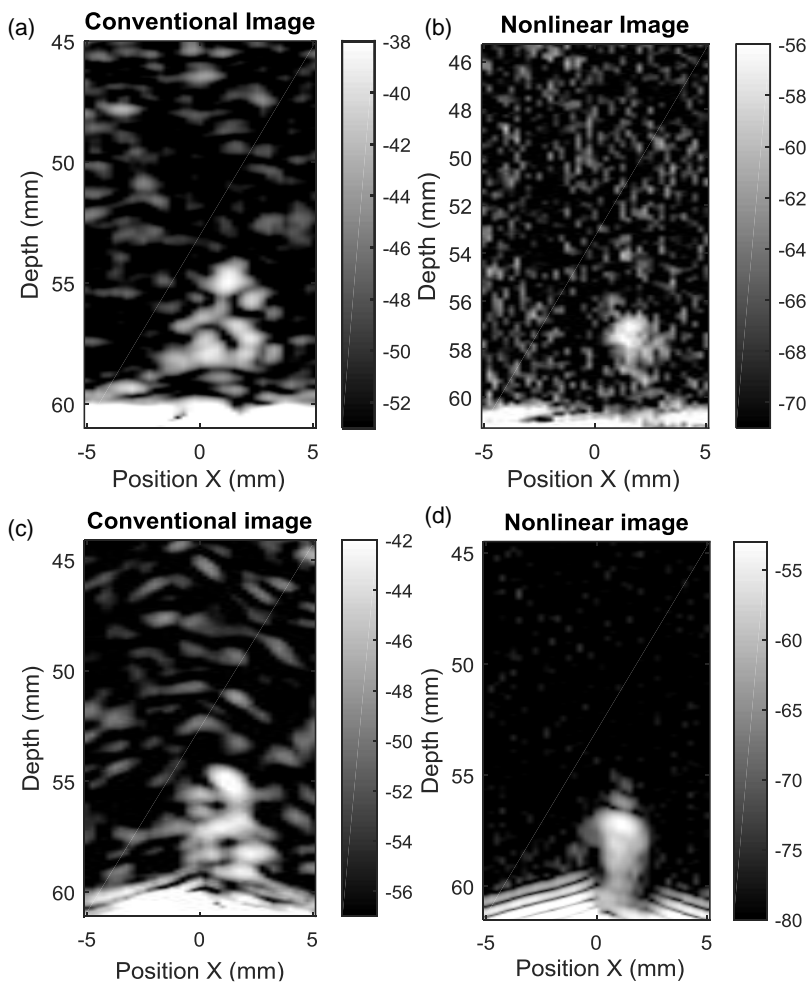

Fig. 5. Conventional and nonlinear images obtained with (a, b) a M2M MultiX++ coupled to an Imasonic probe $(128$ elements at $5 \mathrm{MHz})$ and an AOS-PA (c, d) coupled to an Imasonic probe (64 elements at $5 \mathrm{MHz})$. The dynamic gray level intensity are $15 \mathrm{~dB}$ and $25 \mathrm{~dB}$ for conventional (a, c) and nonlinear (b, d) images respectively. [intensity is in $\mathrm{dB}$ ]

\section{Conclusion.}

In this paper, the AM method routinely used for contrast enhanced medical echography is successfully transposed to NDT field for small cracks inspection. This study shows that it is possible to specifically image a $5.9 \mathrm{~mm}$-deep realistic crack produced by thermal fatigue, with a better contrast than the conventional image. Moreover, conventional and nonlinear images give complementary information indicating open and (partially-) closed part of the crack, respectively. Finally, contrary to most nonlinear methods, the AM method does not require high power voltage to excite the nonlinear scatterers. Furthermore, as the AM method reduces temporal resolution (i.e. frame rate) by a factor of 3 compared to conventional imaging, the method 
can be implemented for real-time inspection on conventional phased array ultrasonic devices. It opens new perspectives for hazard monitoring of civil engineering constructions or for non-destructive testing of metallic structures and composite structures.

\section{Acknowledgements}

This work was supported by EDF R\&D in France.

The authors wish to thank Arnaud Derode and AOSNDT company who lent us the Lecoeur and the AOS-PA multi-channel electronic systems respectively.

*sylvain.haupert@upmc.fr

\section{References}

[1] Zheng YP, Maev RG, Solodov IY. Nonlinear acoustic applications for material characterization: A review. Canadian Journal of Physics 1999;77:927-67.

[2] Ostrovsky LA, Johnson PA. Dynamic nonlinear elasticity in geomaterials. Rivista Del Nuovo Cimento 2001;24:1-46.

[3] Jhang K-Y. Nonlinear ultrasonic techniques for nondestructive assessment of micro damage in material: a review. International Journal of Precision Engineering and Manufacturing 2009;10:123-135.

[4] Meo M, Polimeno U, Zumpano G. Detecting damage in composite material using nonlinear elastic wave spectroscopy methods. Applied Composite Materials 2008;15:115-26.

[5] Van den Abeele K, Le Bas P, Van Damme B, Katkowski T. Quantification of material nonlinearity in relation to microdamage density using nonlinear reverberation spectroscopy: Experimental and theoretical study. The Journal of the Acoustical Society of America 2009;126:963.

[6] Aymerich F, Staszewski W. Experimental Study of Impact-Damage Detection in Composite Laminates using a Cross-Modulation Vibro-Acoustic Technique. Structural Health Monitoring 2010;9:541.

[7] Van den Abeele K, Sutin A, Carmeliet J, Johnson PA. Micro-damage diagnostics using nonlinear elastic wave spectroscopy (NEWS). Ndt \& E International 2001;34:239-48.

[8] Haupert S, Guérard S, Peyrin F, Mitton D, Laugier P. Non Destructive Characterization of Cortical Bone Micro-Damage by Nonlinear Resonant Ultrasound Spectroscopy. PLoS ONE 2014;9:e83599. doi:10.1371/journal.pone.0083599.

[9] Moreschi H, Callé S, Guerard S, Mitton D, Renaud G, Defontaine M. Monitoring trabecular bone microdamage using a dynamic acousto-elastic testing method. Proceedings of the Institution of Mechanical
Engineers, Part H: Journal of Engineering in Medicine 2011;225:1-12.

[10] Duffour P, Morbidini M, Cawley P. A study of the vibro-acoustic modulation technique for the detection of cracks in metals. The Journal of the Acoustical Society of America 2006;119:1463.

[11] Courtney C, Drinkwater B, Neild S, Wilcox P. Factors affecting the ultrasonic intermodulation crack detection technique using bispectral analysis. NDT \& E International 2008;41:223-34.

[12] Zardan JP, Payan C, Garnier V, Salin J. Effect of the presence and size of a localized nonlinear source in concrete. J Acoust Soc Am 2010;128:EL38-42. doi:10.1121/1.3448024.

[13] Donskoy D, Sutin A, Ekimov A. Nonlinear acoustic interaction on contact interfaces and its use for nondestructive testing. Ndt \& E International 2001;34:231-8.

[14] Straka L, Yagodzinskyy Y, Landa M, Hänninen H. Detection of structural damage of aluminum alloy 6082 using elastic wave modulation spectroscopy. NDT \& E International 2008;41:554-63.

[15] Haupert S, Guérard S, Mitton D, Peyrin F, Laugier P. Quantification of nonlinear elasticity for the evaluation of submillimeter crack length in cortical bone. Journal of the Mechanical Behavior of Biomedical Materials 2015;48:210-9. doi:10.1016/j.jmbbm.2015.04.013.

[16] Mezil S, Chigarev N, Tournat V, Gusev V. Two dimensional nonlinear frequency-mixing photoacoustic imaging of a crack and observation of crack phantoms. Journal of Applied Physics 2013;114:174901. doi:10.1063/1.4827180.

[17] Ulrich T, Johnson PA, Guyer RA. Interaction dynamics of elastic waves with a complex nonlinear scatterer through the use of a time reversal mirror. Physical Review Letters 2007;98:104301.

[18] Bas PYL, Ulrich TJ, Anderson BE, Guyer RA, Johnson PA. Probing the interior of a solid volume with time reversal and nonlinear elastic wave spectroscopy. The Journal of the Acoustical Society of America 2011;130:EL258-EL263. doi:10.1121/1.3638926.

[19] Payan C, Ulrich TJ, Bas PYL, Griffa M, Schuetz P, Remillieux MC, et al. Probing material nonlinearity at various depths by time reversal mirrors. Applied Physics Letters 2014;104:144102. doi:10.1063/1.4871094.

[20] Solodov I, Wackerl J, Pfleiderer K, Busse G. Nonlinear self-modulation and subharmonic acoustic spectroscopy for damage detection and location. Applied Physics Letters 2004;84:5386. 
[21] Haupert S, Rivière J, Anderson B, Ohara Y, Ulrich TJ, Johnson P. Optimized Dynamic Acoustoelasticity Applied to Fatigue Damage and Stress Corrosion Cracking. Journal of Nondestructive Evaluation 2014:1-13.

[22] Ohara Y, Mihara T, Sasaki R, Ogata T, Yamamoto S, Kishimoto $\mathrm{Y}$, et al. Imaging of closed cracks using nonlinear response of elastic waves at subharmonic frequency. Applied Physics Letters 2007;90:011902.

[23] Potter JN, Croxford AJ, Wilcox PD. Nonlinear Ultrasonic Phased Array Imaging. Phys Rev Lett 2014;113:144301. doi:10.1103/PhysRevLett.113.144301.

[24] Eckersley RJ, Chin CT, Burns PN. Optimising phase and amplitude modulation schemes for imaging microbubble contrast agents at low acoustic power. Ultrasound in Medicine \& Biology 2005;31:213-9. doi:10.1016/j.ultrasmedbio.2004.10.004.

[25] Burns PN, Wilson SR, Simpson DH. Pulse inversion imaging of liver blood flow: improved method for characterizing focal masses with microbubble contrast. Invest Radiol 2000;35:58-71.

[26] Couture O, Bannouf S, Montaldo G, Aubry J-F, Fink M, Tanter M. Ultrafast Imaging of Ultrasound Contrast Agents. Ultrasound in Medicine \& Biology 2009;35:1908-16.

doi:10.1016/j.ultrasmedbio.2009.05.020.

[27] Bruno C, Gliozzi A, Scalerandi M, Antonaci P. Analysis of elastic nonlinearity using the scaling subtraction method. Physical Review B 2009;79:64108.

[28] Antonaci P, Bruno C, Bocca P, Scalerandi M, Gliozzi A. Nonlinear ultrasonic evaluation of load effects on discontinuities in concrete. Cement and Concrete Research 2010;40:340-6.

[29] Rivière J, Haupert S, Laugier P, Ulrich T, Le Bas PY, Johnson PA. Time reversed elastic nonlinearity diagnostic applied to mock osseointegration monitoring applying two experimental models. The Journal of the Acoustical Society of America 2012;131:1922-7.

[30] Kemppainen M, Virkkunen I, Pitkänen J, Paussu R, Hänninen H. Advanced flaw production method for in-service inspection qualification mock-ups. Nuclear Engineering and Design 2003;224:105-17. doi:10.1016/S0029-5493(03)00078-5.

[31] Broda D, Staszewski WJ, Martowicz A, Uhl T, Silberschmidt VV. Modelling of nonlinear crackwave interactions for damage detection based on ultrasound-A review. Journal of Sound and Vibration 2014;333:1097-1118.

[32] Ohara Y, Yamamoto S, Mihara T, Yamanaka K. Ultrasonic Evaluation of Closed Cracks Using
Subharmonic Phased Array. Jpn J Appl Phys 2008;47:3908. doi:10.1143/JJAP.47.3908. 